\title{
Prediction of uncomplicated pregnancies in obese women: a prospective multicentre study
}

\author{
Matias C. Vieira ${ }^{1,2}$, Sara L. White ${ }^{1}$, Nashita Patel ${ }^{1}$, Paul T. Seed ${ }^{1}$, Annette L. Briley ${ }^{1,3}$, Jane Sandall ${ }^{1}$, Paul Welsh ${ }^{4}$, \\ Naveed Sattar ${ }^{4}$, Scott M. Nelson ${ }^{5}$, Debbie A. Lawlor ${ }^{6,7}$, Lucilla Poston ${ }^{1,3}$, Dharmintra Pasupathy ${ }^{1,3,8^{*}}$ and on behalf \\ of the UPBEAT Consortium
}

\begin{abstract}
Background: All obese pregnant women are considered at equal high risk with respect to complications in pregnancy and birth, and are commonly managed through resource-intensive care pathways. However, the identification of maternal characteristics associated with normal pregnancy outcomes could assist in the management of these pregnancies. The present study aims to identify the factors associated with uncomplicated pregnancy and birth in obese women, and to assess their predictive performance.

Methods: Data form obese women (BMI $\geq 30 \mathrm{~kg} / \mathrm{m}^{2}$ ) with singleton pregnancies included in the UPBEAT trial were used in this analysis. Multivariable logistic regression was used to identify sociodemographic, clinical and biochemical factors at $15^{+0}$ to $18^{+6}$ weeks' gestation associated with uncomplicated pregnancy and birth, defined as delivery of a term live-born infant without antenatal or labour complications. Predictive performance was assessed using area under the receiver operating characteristic curve (AUROC). Internal validation and calibration were also performed. Women were divided into fifths of risk and pregnancy outcomes were compared between groups. Sensitivity, specificity, and positive and negative predictive values were calculated using the upper fifth as the positive screening group.

Results: Amongst 1409 participants (BMI 36.4, SD $4.8 \mathrm{~kg} / \mathrm{m}^{2}$ ), the prevalence of uncomplicated pregnancy and birth was 36\% (505/1409). Multiparity and increased plasma adiponectin, maternal age, systolic blood pressure and $\mathrm{HbA1C}$ were independently associated with uncomplicated pregnancy and birth. These factors achieved an AUROC of $0.72(0.68-0.76)$ and the model was well calibrated. Prevalence of gestational diabetes, preeclampsia and other hypertensive disorders, preterm birth, and postpartum haemorrhage decreased whereas spontaneous vaginal delivery increased across the fifths of increasing predicted risk of uncomplicated pregnancy and birth. Sensitivity, specificity, and positive and negative predictive values were $38 \%, 89 \%, 63 \%$ and $74 \%$, respectively. A simpler model including clinical factors only (no biomarkers) achieved an AUROC of 0.68 (0.65-0.71), with sensitivity, specificity, and positive and negative predictive values of 31\%, $86 \%, 56 \%$ and $69 \%$, respectively.

Conclusion: Clinical factors and biomarkers can be used to help stratify pregnancy and delivery risk amongst obese pregnant women. Further studies are needed to explore alternative pathways of care for obese women demonstrating different risk profiles for uncomplicated pregnancy and birth.
\end{abstract}

Keywords: Obesity, Prediction, Uncomplicated pregnancy, Birth, Pregnancy outcome, Risk stratification

\footnotetext{
* Correspondence: dharmintra.pasupathy@kcl.ac.uk

'Department of Women and Children's Health, School of Life Course

Sciences, Faculty of Life Sciences and Medicine, King's College London,

London SE1 7EH, UK

${ }^{3} \mathrm{NIHR}$ Biomedical Research Centre at Guy's and St Thomas' NHS Foundation

Trust and King's College London, London SE1 7EH, UK

Full list of author information is available at the end of the article
} 


\section{Background}

Maternal obesity is associated with increased risk of maternal and perinatal mortality and morbidity, including gestational diabetes mellitus (GDM), preeclampsia and birth complications [1]. In common with global trends of obesity in the non-pregnant population, the prevalence of maternal obesity is increasing, with one in four women of reproductive age in the UK being classed as obese [1, 2]. The NHS costs of care and management of pregnancy complications in obese women have been estimated at an additional $£ 1171$ (37\%) per pregnancy compared to women of normal body mass index (BMI) [3].

A key strategy of the UK 2016 Maternity Transformation Programme is the evidence-based provision of antenatal care that recognises different levels of risk of adverse outcomes between different groups of women [4]. The UK National Maternity Review, 'Better Births' [5], notes that a simple dichotomy of 'high risk' or 'no risk' is too simplistic, and women are requesting more detailed information about their risks. In this context, defining all obese pregnant women as 'high risk' may not always ensure provision of the most effective care nor a choice of care pathway. We have previously suggested that maternity care might benefit from a shift away from the current focus on complications towards the pursuit of uncomplicated pregnancy [6]. The identification of maternal characteristics associated with normal pregnancy outcomes in obese women and the combining of these factors in a risk stratification algorithm could help inform women's decision-making with regards to the management of their pregnancies as well as the provision and allocation of resources for pregnancy care for obese women.

The aim of the present study is to identify factors in the early second trimester associated with subsequent uncomplicated pregnancy and birth in obese women and to assess their predictive performance.

\section{Methods}

The UK Pregnancies Better Eating and Activity Trial (UPBEAT; ISCRTN89971375) was a multicentre, randomised controlled trial of a complex behavioural intervention of diet and physical activity advice versus standard antenatal care in obese pregnant women to prevent GDM and delivery of large for gestational age infants. The study involved eight centres located in London (three centres), Bradford, Glasgow, Manchester, Newcastle, and Sunderland. Regulatory approvals were obtained from the UK research ethics committee (UK integrated research application system, reference 09/H0802/5) and local research and development departments in participating centres. All women provided written informed consent prior to entering the study.
UPBEAT recruited 1555 obese women (BMI $\geq 30 \mathrm{~kg}$ / $\mathrm{m}^{2}$ ), aged 16 years or older, and with a singleton pregnancy between $15^{+0}$ and $18^{+6}$ weeks' gestation. Women were recruited from March 2009 to June 2014. Exclusion criteria were multiple pregnancy, current use of metformin, unwilling or unable to provide written informed consent, or underlying disorders (including a prepregnancy diagnosis of essential hypertension, diabetes, renal disease, systemic lupus erythematosus, antiphospholipid syndrome, sickle cell disease, thalassaemia, coeliac disease, thyroid disease or current psychosis). Extensive data were collected on sociodemographic and clinical characteristics, and anthropometric measures and blood samples were also obtained. Women were followed up at $27^{+0}$ to $28^{+6}$ and $34^{+0}$ to $35^{+6}$ weeks' gestation, at delivery and at 6 months postpartum [7, 8]. Except for the UPBEAT behavioural intervention, routine antenatal care according to UK and local practice was provided to all women in the study.

For the purpose of this study, women with missing information for pregnancy outcomes were excluded. The UPBEAT intervention was not associated with an effect on the primary outcomes or any relevant pregnancy outcome [8], and therefore the study population was treated as a cohort for the purpose of this analysis [9].

\section{Outcomes}

The outcome of interest was an uncomplicated pregnancy and birth defined in this study as a pregnancy without any antenatal or labour complications following recruitment at $15^{+0}$ to $18^{+6}$ weeks' gestation. Antenatal complications were late miscarriage, preterm birth (before 37 weeks' gestation), GDM, preeclampsia, hypertensive disorders of pregnancy, antepartum haemorrhage, placental abruption, venous thromboembolism, delivery of a small for gestational age infant (birthweight below the 10th customised centile [10]) and stillbirth (after 20 weeks' gestation). Labour complications were instrumental vaginal birth, emergency caesarean section, postpartum haemorrhage (above $1000 \mathrm{~mL}$ ), Apgar score $<7$ at 5 minutes, neonatal intensive care unit admission and neonatal death. Preeclampsia was defined as blood pressure $\geq 140 / 90 \mathrm{mmHg}$ associated with proteinuria of $\geq 300 \mathrm{mg} / 24 \mathrm{~h}$, or a spot urine protein:creatinine ratio of $\geq 30 \mathrm{mg} / \mathrm{mmol}$ creatinine, or urine dipstick protein $\geq 2+[11]$, and a GDM diagnosis was based on a $75 \mathrm{~g}$ oral glucose tolerance test (OGTT) using the International Association of Diabetes and Pregnancy Study Groups (IADPSG) criteria [12]. Universal OGTT was part of the study protocol but, where not performed, a clinical diagnosis of GDM was used.

Potential predictors of uncomplicated pregnancy and birth Factors from early pregnancy $\left(15^{+0}\right.$ to $18^{+6}$ weeks' gestation) were selected on the basis of known associations 
with one or more pregnancy complications. The sociodemographic characteristics and clinical factors explored were maternal age, ethnicity (white, black, Asian or other), adjusted index of multiple deprivation, BMI, parity, previous history of GDM or preeclampsia, smoking, history of threatened miscarriage in the index pregnancy, systolic blood pressure (SBP) and maternal anthropometry. Maternal anthropometric measures included were mid-arm circumference and sum of skinfold thickness (triceps, biceps, subscapular and suprailiac). Blood pressure was recorded using the pregnancy validated Microlife BP3BT0-A blood pressure monitor (Microlife, Widnau, Switzerland) and maternal skinfold thicknesses (triceps, biceps, suprailiac and subscapular) were measured in triplicate, using Harpenden skinfold Calipers (Holtain Ltd, Felin-y-Gigfran, Crosswell, UK) [13].

A total of 19 selected biomarkers measured in blood samples obtained at $15^{+0}$ to $18^{+6}$ weeks' gestation were assessed. The biomarkers measured in blood samples were markers of glucose homeostasis (haemoglobin A1c (HbA1c), fructosamine, insulin and C-peptide), adipokines (adiponectin and leptin), inflammatory and endothelial markers (interleukin-6, high sensitivity C-reactive protein, and t-PA antigen), lipids (triglycerides, total cholesterol, LDL cholesterol and HDL cholesterol), liverassociated markers (aspartate aminotransferase, alanine aminotransferase, gamma-glutamyl transferase (gGT), sex hormone binding globulin and ferritin) and vitamin D. Analytical methods for these biomarkers are shown in Additional file 1.

\section{Statistical analysis}

All participants with complete pregnancy outcome data were included in the analysis. To ensure that the study population was representative of the overall UPBEAT participants we compared demographic characteristics between women included and excluded from this analysis. Missing data on potential predictors were minimal $(<2 \%)$, except for biomarkers, as blood samples were not available for $27 \%$ of women (286/1409). Of those with blood samples taken, biomarker missing data were minimal $(<2 \%)$, except for HbA1c $(5.7 \%$; 59/1023). Biomarkers were assessed for normality and variation with gestational age. None of the biomarkers showed variation with gestational age at measurement and biomarkers with highly skewed distributions were transformed into $\log _{2}$ (insulin, C-peptide, adiponectin, leptin, interleukin-6, high sensitivity C-reactive protein, $\mathrm{t}-\mathrm{PA}$ antigen, triglycerides, HDL cholesterol, aspartate aminotransferase, alanine aminotransferase, gGT, ferritin and vitamin D), so that odds ratios (ORs) showed the effect associated with doubling the concentration. Ethnicity was transformed into a binary (white and non-white) variable for analysis to reduce the degrees of freedom. The index of multiple deprivation was categorised into fifths for a clearer interpretation. Continuous data were summarised by mean (standard deviation) and categorical data reported as number (percentage). Comparisons between groups were performed using a $t$-test or $\chi^{2}$ test, as appropriate. Univariable logistic regression was used to estimate the effect size for each factor. Clinical factors with a $P$ value less than 0.05 in group comparison were included in multivariable analyses. Biomarkers with a statistically significant association following Bonferroni correction for 19 tests $(P<$ 0.0026 ) were included in multivariable analyses.

Backward stepwise logistic regression was then used to identify factors that were independently associated with uncomplicated pregnancies and birth [14]. One multivariable model was developed to include only clinical factors, and a second model included both clinical factors and biomarkers. A sensitivity analysis was performed to assess if the reduction in the sample size for the assessment of biomarkers had any effect on the size of the association between clinical factors and outcome. The outcome of this study was a normal event instead of an adverse outcome, factors with an OR smaller than one were considered predictors of an adverse outcome, whereas those with an OR greater than one were considered to predict increased likelihood of an uncomplicated pregnancy. The overall accuracy of the final multivariable model to discriminate between uncomplicated and complicated pregnancy was assessed using the area under the receiver operating characteristic curve (AUROC). Internal cross-validation using a 10-fold split in the dataset was performed [15]. Calibration was assessed by comparing observed to predicted levels of uncomplicated pregnancy and birth within tenths of predicted risk and a Hosmer-Lemeshow test was performed. Women were then divided into fifths of predicted risk and pregnancy outcomes were compared between the fifths. Finally, the upper fifth of predicted levels of uncomplicated pregnancy and birth was considered a positive screening group and diagnostic test performance was assessed (sensitivity, specificity, positive predictive value and negative predictive value). All statistical analyses were performed using Stata software, version 14.1 (StataCorp, College Station, Texas). This study was reported in accordance with the TRIPOD guideline for reporting the development of multivariable prediction models [16].

\section{Results}

The UPBEAT trial recruited 1555 obese women between $15^{+0}$ and $18^{+6}$ weeks' gestation. In this analysis, the study population comprised 1409 women with complete outcome data (Fig. 1) and there were no obvious differences in demographic characteristics between our study population and UPBEAT participants excluded from this 


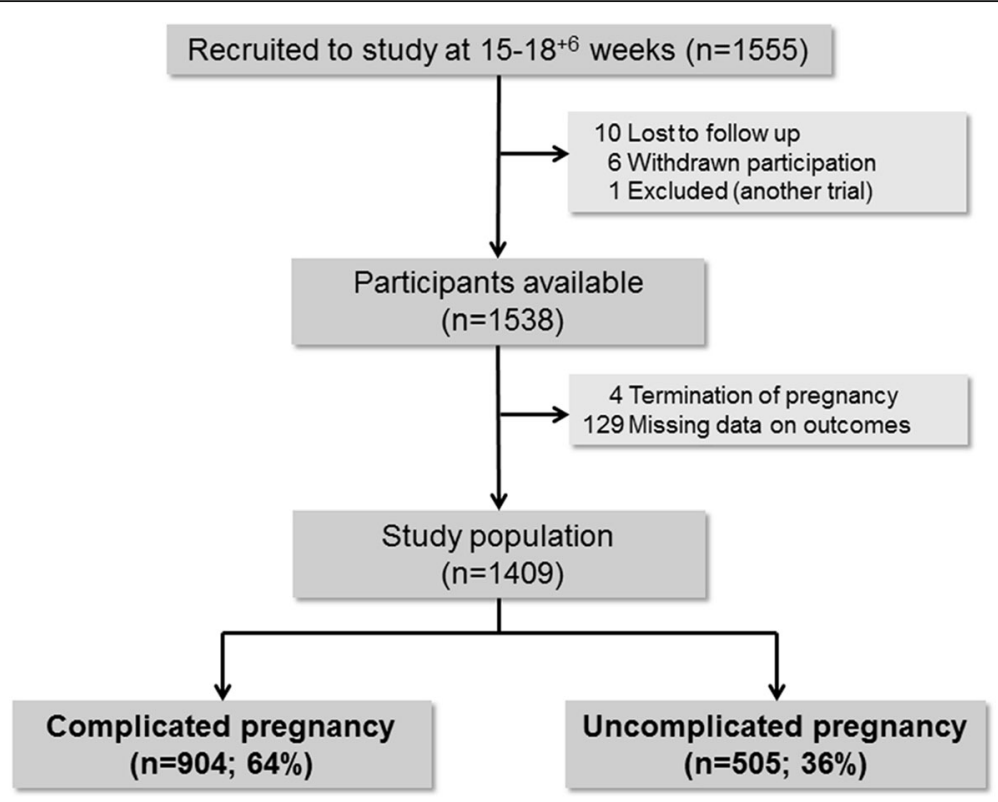

Fig. 1 Study population

analysis. Additional file 2 describes these comparisons. The prevalence of uncomplicated pregnancy and birth in the study population was $36 \%(505 / 1409)$.

Amongst women with complications, 38\% (342/904) had antenatal complications alone, 32\% (287/904) had labour complications alone and 30\% (275/904) experienced both. Women who experienced both antenatal and labour complications accounted for 20\% (275/1409) of the whole study population (Additional file 3 shows this distribution). The prevalence of each pregnancy complication is described in Table 1. GDM was the leading antenatal complication and emergency caesarean section was the most prevalent labour complication.

The sociodemographic characteristics of women with and without complications in pregnancy are described in Table 2, and the descriptive statistics for potential predictors of uncomplicated pregnancy and birth are shown in Additional file 4.

In univariable analyses, multiparity was associated with increased odds of an uncomplicated pregnancy and birth, and factors associated with a reduced odds were higher maternal age, SBP, BMI, mid-arm circumference and sum of skinfolds (Table 3). A previous history of either GDM or preeclampsia was also associated with reduced odds of uncomplicated pregnancy and birth. Higher concentrations of adiponectin were associated with greater odds of uncomplicated pregnancy and birth, while reduced odds were observed for higher HbA1c, insulin, sex hormone binding globulin and gGT. In multivariable analysis, multiparity, SBP, adiponectin and HbA1c were independently associated with uncomplicated pregnancy and birth (Table 3). Using these factors from multivariable analysis, the algorithm achieved an AUROC of 0.73 (0.69-0.76) (Table 3). Model performance was confirmed by internal validation with an AUROC of 0.72 (0.68-0.76). The model also had good calibration as demonstrated by the similarity of predicted and observed proportions of uncomplicated pregnancies and births across tenths of predicted risk (Fig. 2; Hosmer-Lemeshow $P=0.54$ ).

Table 1 Prevalence of each pregnancy complication

\begin{tabular}{|c|c|c|}
\hline & $N(n=1409)$ & Percent \\
\hline \multicolumn{3}{|l|}{ Antenatal complications } \\
\hline Miscarriage & 8 & 0.6 \\
\hline Preterm birth & 77 & 5.5 \\
\hline Gestational diabetes & 334 & 23.7 \\
\hline Preeclampsia & 53 & 3.8 \\
\hline Other hypertensive disorders & 83 & 5.9 \\
\hline Antepartum haemorrhage & 54 & 3.8 \\
\hline Placental abruption & 9 & 0.6 \\
\hline Venous thromboembolism & 4 & 0.3 \\
\hline Small for gestational age & 162 & 11.5 \\
\hline Stillbirth & 7 & 0.5 \\
\hline \multicolumn{3}{|l|}{ Labour complications } \\
\hline Instrumental delivery & 165 & 11.7 \\
\hline Emergency caesarean section & 237 & 16.8 \\
\hline Postpartum haemorrhage & 186 & 13.2 \\
\hline Apgar $<7$ at 5 min & 28 & 2.0 \\
\hline Neonatal intensive care & 113 & 8.0 \\
\hline Neonatal death & 4 & 0.3 \\
\hline
\end{tabular}


Table 2 Sociodemographic characteristics in pregnancies with and without complications in pregnancy and/or birth

\begin{tabular}{lll}
\hline & $\begin{array}{l}\text { Complicated } \\
\text { pregnancy, } \\
\text { Mean (SD) or n (\%) }\end{array}$ & $\begin{array}{l}\text { Uncomplicated } \\
\text { pregnancy, } \\
\text { Mean (SD) or n (\%) }\end{array}$ \\
\hline Age & $30.6(5.4)$ & $30.0(5.7)$ \\
Body mass index & $36.6(5.2)$ & $35.9(4.1)$ \\
Ethnicity & & \\
White & $567 / 904(62.7)$ & $319 / 505(63.2)$ \\
Black & $224 / 904(24.8)$ & $132 / 505(26.1)$ \\
Asian & $55 / 904(6.1)$ & $31 / 505(6.1)$ \\
Other & $58 / 904(6.4)$ & $23 / 505(4.6)$ \\
Multiparous & $416 / 904(46.0)$ & $370 / 505(73.3)$ \\
Previous history of GDM & $22 / 416(5.3)$ & $6 / 370(1.6)$ \\
Previous history of PE & $37 / 416(8.9)$ & $25 / 370(6.8)$ \\
IMD fifths & & $19 / 504(3.8)$ \\
1 (least deprived) & $33 / 899(3.7)$ & $32 / 504(6.4)$ \\
2 & $62 / 899(6.9)$ & $61 / 504(12.1)$ \\
3 & $95 / 899(10.6)$ & $164 / 504(32.5)$ \\
4 & $316 / 899(35.2)$ & $228 / 504(45.2)$ \\
5 (most deprived) & $393 / 899(43.7)$ & $32 / 505(6.3)$ \\
Current smoker & $66 / 904(7.3)$ & d \\
\hline
\end{tabular}

GDM gestational diabetes mellitus, IMD index of multiple deprivation, PE preeclampsia
Women were then divided in fifths of predicted risk and the prevalence of each adverse outcome in the fifths is described in Table 4. With an increasing predicted chance of uncomplicated pregnancies and birth, a trend of reduced prevalence of antenatal (preterm delivery, gestational diabetes, preeclampsia and other hypertensive disorders) and labour complications (caesarean section and postpartum haemorrhage) were observed. Sensitivity, specificity, and positive and negative predictive value of the upper (5th) fifth (compared with all other women) for an uncomplicated pregnancy and birth were $38 \%(32-43 \%), 89 \%$ (86-91\%), 63\% (55-70\%) and $74 \%$ (71-77\%), respectively. Positive likelihood ratio and negative likelihood ratio were also calculated (3.3 and 0.7 , respectively).

The model including only clinical factors (AUROC of $0.68,0.65-0.71$ ) was also used to explore the prevalence of each adverse outcome in each fifth of predicted risk (Table 5). Diagnostic test performance was also calculated for this model. Sensitivity, specificity, and positive and negative predictive values of the upper fifth were $31 \%(27-36 \%), 86 \%$ (84-89\%), 56\% (50-62\%) and 69\% (66-72\%), respectively. The positive and negative likelihood ratios were 2.3 and 0.8 , respectively. Additional file 5 describes the fifths of predicted risk for an alternative model including clinical factors and HbA1c (AUROC $0.71,0.66-0.75$ ). This post-hoc analysis was performed as $\mathrm{HbA1c}$ is readily available in clinical practice in most countries, while adiponectin is more often available in research settings. Sensitivity, specificity, and positive and

Table 3 Factors associated with uncomplicated pregnancies and birth, and their predictive performance

\begin{tabular}{|c|c|c|c|}
\hline & $\begin{array}{l}\text { Univariable }{ }^{a} \\
\text { OR }(95 \% \mathrm{Cl})\end{array}$ & $\begin{array}{l}\text { Multivariable (Clinical factors only), } \\
\text { OR }(95 \% \mathrm{Cl}), n=1370\end{array}$ & $\begin{array}{l}\text { Multivariable (All factors), } \\
\text { OR }(95 \% \mathrm{Cl}), n=907\end{array}$ \\
\hline Maternal age (per 5 year), $n=1409$ & $0.90(0.82-1.00)$ & $0.79(0.71-0.88)$ & $0.81(0.71-0.93)$ \\
\hline Multiparous, $n=1409$ & $3.22(2.54-4.07)$ & $3.54(2.75-4.55)$ & $4.23(3.04-5.88)$ \\
\hline Previous history of GDM or $\mathrm{PE}, n=786$ & $0.58(0.36-0.91)$ & & \\
\hline $\mathrm{SBP}($ per $10 \mathrm{mmHg}), n=1387$ & $0.77(0.69-0.85)$ & $0.82(0.73-0.91)$ & $0.84(0.73-0.97)$ \\
\hline BMI $\left(\right.$ per $\left.5 \mathrm{~kg} / \mathrm{m}^{2}\right), n=1409$ & $0.84(0.74-0.94)$ & $0.85(0.74-0.97)$ & \\
\hline Mid-arm circumference (per $1 \mathrm{~cm}) n=1398$ & $0.96(0.94-0.99)$ & & \\
\hline Sum of skinfolds (per $1 \mathrm{~cm}$ ), $n=1390$ & $0.94(0.91-0.98)$ & & \\
\hline \multicolumn{4}{|l|}{ Biomarkers } \\
\hline $\mathrm{HbA} 1 \mathrm{c}($ per $1 \mathrm{mmol} / \mathrm{mol}), n=949$ & $0.90(0.87-0.94)$ & & $0.90(0.86-0.94)$ \\
\hline Insulin (per doubling, $\log _{2}$ of $\mathrm{mU} / \mathrm{L}$ ), $n=1017$ & $0.86(0.78-0.94)$ & & \\
\hline Adiponectin (per doubling, $\log _{2}$ of $\mu \mathrm{g} / \mathrm{mL}$ ), $n=1013$ & $1.34(1.16-1.56)$ & & $1.40(1.18-1.66)$ \\
\hline gGT (per doubling, $\log _{2}$ of $U / L$ ), $n=1015$ & $0.78(0.67-0.89)$ & & \\
\hline SHBG (per $1 \mathrm{nmol} / \mathrm{L}$ ), $n=1004$ & $1.00(1.00-1.00)$ & & \\
\hline AUROC & & $0.69(0.66-0.71)$ & $0.73(0.69-0.76)$ \\
\hline Internal validation AUROC & & $0.68(0.65-0.71)$ & $0.72(0.68-0.76)$ \\
\hline
\end{tabular}

${ }^{\mathrm{a}}$ In univariable analyses numbers vary depending on missing data and are given in the first column

$A U R O C$ area under the receiver operating characteristic curve, $B M I$ body mass index, GDM gestational diabetes mellitus, $g G T$ gamma-glutamyl transferase, $H b A 1 c$ haemoglobin A1c, PE preeclampsia, SBP systolic blood pressure, SHBG sex hormone binding globulin 


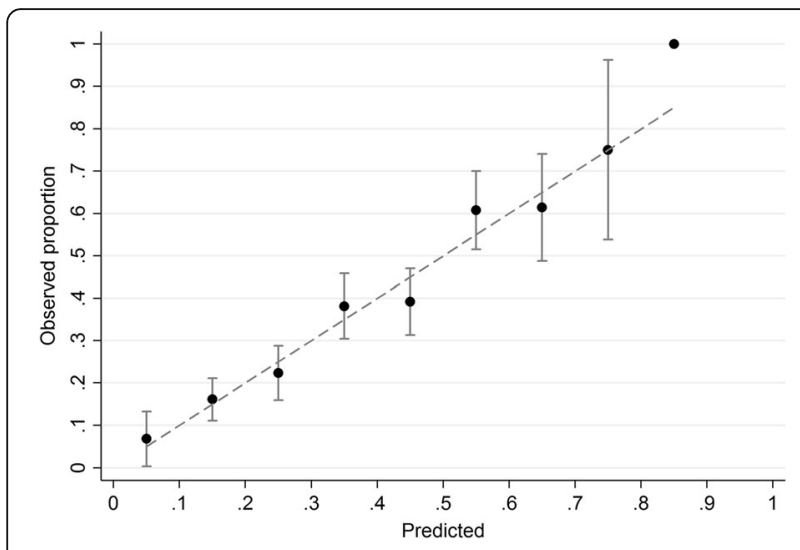

Fig. 2 Calibration of the full prediction model for uncomplicated pregnancies in obese women negative predictive values of the upper fifth using this alternative model were 33\% (29-40\%), 87\% (84-90\%), 57\% (49-64\%) and 72\% (69-76\%), respectively.

In sensitivity analyses, we found that the point estimates for the associations of clinical predictors with uncomplicated pregnancy and birth were broadly similar in the group with maximal data and those who were excluded because of missing data. Additional file 6 describes these results.

\section{Discussion}

Over one-third of this contemporary, multi-ethnic, inner city population of obese pregnant women had an uncomplicated pregnancy and birth. Nevertheless, this also highlights the extent to which obesity is an important risk factor for pregnancy complications. Combining clinical factors and biomarkers, we identified five independent predictors in early pregnancy (at $15^{+0}$ to $18^{+6}$ weeks' gestation) that were associated with an uncomplicated

Table 4 Pregnancy outcomes according to predicted chance in fifths of uncomplicated pregnancy and birth using the full model

\begin{tabular}{|c|c|c|c|c|c|c|}
\hline & $\begin{array}{l}\text { Least likely to have } \\
\text { an uncomplicated } \\
\text { pregnancy and birth }\end{array}$ & & & $\begin{array}{l}\text { Most likely to have } \\
\text { an uncomplicated } \\
\text { pregnancy and birth }\end{array}$ & & \\
\hline & $\begin{array}{l}\text { 1st fifth } \\
(n=181)\end{array}$ & $\begin{array}{l}\text { 2nd fifth } \\
(n=181)\end{array}$ & $\begin{array}{l}\text { 3rd fifth } \\
(n=182)\end{array}$ & $\begin{array}{l}4 \text { th fifth } \\
(n=181)\end{array}$ & $\begin{array}{l}\text { 5th fifth } \\
(n=182)\end{array}$ & $P$ value \\
\hline Uncomplicated pregnancy (\%) & $22(12.2)$ & $34(18.8)$ & $58(31.9)$ & $75(41.4)$ & $114(62.6)$ & $<0.001$ \\
\hline \multicolumn{7}{|l|}{ Antenatal outcomes (\%) } \\
\hline Miscarriage $^{a}$ & $2(1.1)$ & $2(1.1)$ & $1(0.5)$ & $3(1.7)$ & $0(0)$ & 0.51 \\
\hline Preterm delivery & $11(6.1)$ & $16(8.9)$ & $6(3.3)$ & $7(3.9)$ & $5(2.7)$ & 0.04 \\
\hline Gestational diabetes & $77(43)$ & $50(27.9)$ & $57(31.5)$ & $35(19.7)$ & $29(15.9)$ & $<0.001$ \\
\hline Preeclampsia & $12(6.7)$ & $13(7.3)$ & $6(3.3)$ & $3(1.7)$ & $6(3.3)$ & 0.04 \\
\hline Other hypertensive disorders & $16(8.9)$ & $12(6.7)$ & $13(7.2)$ & $9(5.1)$ & $2(1.1)$ & 0.02 \\
\hline Antepartum haemorrhage & $11(6.1)$ & $4(2.2)$ & $8(4.4)$ & $12(6.7)$ & $3(1.6)$ & 0.06 \\
\hline Placental abruption & $2(1.1)$ & $0(0)$ & $1(0.6)$ & $1(0.6)$ & $2(1.1)$ & 0.68 \\
\hline Venous thromboembolism & $0(0)$ & $1(0.6)$ & $0(0)$ & $0(0)$ & $0(0)$ & 0.40 \\
\hline Small for gestational age & $26(14.5)$ & $31(17.3)$ & $18(9.9)$ & $18(10.1)$ & $18(9.9)$ & 0.10 \\
\hline Large for gestational age & $16(8.9)$ & $11(6.1)$ & $18(9.9)$ & $17(9.6)$ & $18(9.9)$ & 0.69 \\
\hline Stillbirth & $2(1.1)$ & $0(0)$ & $1(0.6)$ & $1(0.6)$ & $1(0.5)$ & 0.73 \\
\hline \multicolumn{7}{|l|}{ Labour outcomes (\%) } \\
\hline Induction of labour & $95(52.0)$ & $76(42.5)$ & $60(33.1)$ & $45(25.3)$ & $51(28.0)$ & $<0.001$ \\
\hline Elective caesarean section & $41(22.9)$ & $22(12.3)$ & $34(18.8)$ & $41(23.0)$ & $44(24.2)$ & 0.03 \\
\hline \multicolumn{7}{|l|}{ Mode of delivery (in labour) } \\
\hline Spontaneous vaginal & $56(40.6)$ & $76(48.4)$ & $94(63.9)$ & $105(76.6)$ & $127(92.0)$ & $<0.001$ \\
\hline Instrumental delivery & 27 (19.6) & $35(22.3)$ & $21(14.3)$ & $16(11.7)$ & $3(2.2)$ & $<0.001$ \\
\hline Emergency CS & $55(39.9)$ & $46(29.3)$ & $32(21.8)$ & $16(11.7)$ & $8(5.8)$ & $<0.001$ \\
\hline Postpartum haemorrhage & $44(24.6)$ & $28(15.6)$ & $27(14.9)$ & $20(11.2)$ & $16(8.8)$ & $<0.001$ \\
\hline Apgar $<7$ at 5 min & $6(3.4)$ & $4(2.2)$ & $2(1.1)$ & $4(2.2)$ & $3(1.6)$ & 0.66 \\
\hline NICU admission & 19 (10.6) & $20(11.2)$ & $15(8.3)$ & $12(6.7)$ & $10(5.5)$ & 0.24 \\
\hline Neonatal death & $1(0.6)$ & $1(0.6)$ & $0(0)$ & $0(0)$ & $1(0.5)$ & 0.74 \\
\hline
\end{tabular}

${ }^{a}$ Women with miscarriage were not included in the analysis of other outcomes

CS caesarean section, NICU neonatal intensive care unit 
Table 5 Pregnancy outcomes according to predicted chance in fifths of uncomplicated pregnancy and birth using the clinical model

\begin{tabular}{|c|c|c|c|c|c|c|}
\hline & $\begin{array}{l}\text { Least likely to have } \\
\text { an uncomplicated } \\
\text { pregnancy and birth }\end{array}$ & & & $\begin{array}{l}\text { Most likely to have } \\
\text { an uncomplicated } \\
\text { pregnancy and birth }\end{array}$ & & \\
\hline & $\begin{array}{l}\text { 1st fifth } \\
(n=181)\end{array}$ & $\begin{array}{l}\text { 2nd fifth } \\
(n=181)\end{array}$ & $\begin{array}{l}\text { 3rd fifth } \\
(n=182)\end{array}$ & $\begin{array}{l}\text { 4th fifth } \\
(n=181)\end{array}$ & $\begin{array}{l}\text { 5th fifth } \\
(n=182)\end{array}$ & $P$ value \\
\hline Uncomplicated pregnancy (\%) & $47(17.2)$ & $67(24.5)$ & $98(35.8)$ & $126(46)$ & $154(56.2)$ & $<0.001$ \\
\hline \multicolumn{7}{|l|}{ Antenatal outcomes (\%) } \\
\hline Miscarriage $^{a}$ & $2(0.7)$ & $1(0.4)$ & $2(0.7)$ & $2(0.7)$ & $1(0.4)$ & 0.94 \\
\hline Preterm delivery & $13(4.8)$ & $23(8.4)$ & $8(2.9)$ & $17(6.3)$ & $14(5.1)$ & 0.07 \\
\hline Gestational diabetes & $82(30.1)$ & $59(21.6)$ & $73(26.8)$ & $71(26.1)$ & $39(14.3)$ & $<0.001$ \\
\hline Preeclampsia & $16(5.9)$ & $17(6.2)$ & $8(2.9)$ & $6(2.2)$ & $4(1.5)$ & 0.006 \\
\hline Other hypertensive disorders & $37(13.6)$ & $9(3.3)$ & $19(7.0)$ & $12(4.4)$ & $5(1.8)$ & $<0.001$ \\
\hline Antepartum haemorrhage & $15(5.5)$ & $8(2.9)$ & $9(3.3)$ & $14(5.1)$ & $7(2.6)$ & 0.26 \\
\hline Placental abruption & $2(0.7)$ & $2(0.7)$ & $2(0.7)$ & $3(1.1)$ & $0(0)$ & 0.61 \\
\hline Venous thromboembolism & $2(0.7)$ & $1(0.4)$ & $0(0)$ & $0(0)$ & $0(0)$ & 0.25 \\
\hline Small for gestational age & $34(12.5)$ & $43(15.8)$ & $27(9.9)$ & $27(9.9)$ & $27(9.9)$ & 0.13 \\
\hline Large for gestational age & $22(8.1)$ & $19(7.0)$ & $25(9.2)$ & $30(11.0)$ & $24(8.8)$ & 0.55 \\
\hline Stillbirth & $2(0.7)$ & $2(0.7)$ & $2(0.7)$ & $1(0.4)$ & $0(0)$ & 0.68 \\
\hline \multicolumn{7}{|l|}{ Labour outcomes (\%) } \\
\hline Induction of labour & $145(53.3)$ & $107(39.2)$ & $92(33.8)$ & $72(26.5)$ & $64(23.4)$ & $<0.001$ \\
\hline Elective caesarean section & $47(17.3)$ & $33(12.1)$ & $64(23.5)$ & $70(25.7)$ & $46(16.8)$ & $<0.001$ \\
\hline \multicolumn{7}{|l|}{ Mode of delivery (in labour) } \\
\hline Spontaneous vaginal & $88(39.1)$ & $113(47.1)$ & $136(65.4)$ & $169(83.7)$ & $204(89.9)$ & $<0.001$ \\
\hline Instrumental delivery & $55(24.4)$ & $59(24.6)$ & $25(12.0)$ & $14(6.9)$ & $7(3.1)$ & $<0.001$ \\
\hline Emergency CS & $82(36.4)$ & $68(28.3)$ & $47(22.6)$ & $19(9.4)$ & $16(7.0)$ & $<0.001$ \\
\hline Postpartum haemorrhage & $57(21)$ & $39(14.3)$ & $32(11.8)$ & $27(9.9)$ & $23(8.4)$ & $<0.001$ \\
\hline Apgar $<7$ at $5 \mathrm{~min}$ & $10(3.7)$ & $5(1.8)$ & $5(1.8)$ & $5(1.8)$ & $2(0.7)$ & 0.18 \\
\hline NICU admission & $29(10.7)$ & $23(8.4)$ & $19(7.0)$ & $22(8.1)$ & $18(6.6)$ & 0.45 \\
\hline Neonatal death & $0(0)$ & $1(0.4)$ & $0(0)$ & $3(1.1)$ & $0(0)$ & 0.07 \\
\hline
\end{tabular}

${ }^{a}$ Women with miscarriage were not included in the analysis of other outcomes CS caesarean section, NICU neonatal intensive care unit

pregnancy and birth, namely multiparity, lower maternal age, SBP and HbA1c levels, and higher adiponectin levels. In combination, these achieved an AUROC of 0.72 (0.680.76 ) for the prediction of an uncomplicated pregnancy and birth. The use of the upper fifth of predicted risk of uncomplicated pregnancy and birth as the screen positive group provided a sensitivity of $38 \%$ and a specificity of $89 \%$.

When considering the individual pregnancy complications, the most prevalent during the antenatal period and labour were GDM and emergency caesarean section, respectively. We found that the prevalence of GDM, preeclampsia and other hypertensive disorders, preterm birth, and postpartum haemorrhage decreased and spontaneous vaginal delivery increased across the five groups defined by increasing predicted chance of uncomplicated pregnancy and birth. There was no strong evidence of any difference in miscarriage, antepartum haemorrhage, placental abruption, venous thrombus-embolism, stillbirth, low Apgar score, admission to neonatal intensive care or neonatal death across the five groups, but the numbers in each were few. In general, however, the prevalence of these adverse events was lowest in the group with highest prediction of uncomplicated pregnancy and birth. This suggests that our approach of using one composite outcome is appropriate as those women predicted to have uncomplicated pregnancy and birth were also those shown to have a lower risk for individual adverse outcomes.

To our knowledge, there has been no previous study addressing a composite outcome of uncomplicated pregnancy and birth in obese women. Our previous study explored the factors associated with uncomplicated pregnancy amongst nulliparous women of any BMI [6]. Approximately $61 \%$ of nulliparous women unselected for BMI had uncomplicated pregnancies, compared to $38 \%$ in the present cohort of women with obesity. Although we would expect lower rates of uncomplicated pregnancy and birth outcomes in obese 
women, this difference may be partly attributed to the definition of uncomplicated pregnancy and birth, which varied between studies (instrumental delivery and emergency caesarean section were only considered a pregnancy complication in the present study). Despite the different populations, lower maternal age, SBP and BMI were associated with uncomplicated pregnancy in both studies.

Despite being a widely recognised major risk factor for pregnancy complications [1], the recent World Health Organization recommendations on antenatal care as well as current antenatal guidelines in the UK do not provide specific recommendations for the management of pregnancy in obese women $[17,18]$. The American College of Obstetrics and Gynaecology has published a relevant practice bulletin, but this provides recommendations for all obese women and does not offer stratification of care in this large group [19]. We have shown that it is possible to identify different risk groups amongst obese women, with good discrimination and calibration. Ultimately, we hope that this approach might help improve the management of obese pregnant women. Women with the highest prediction of uncomplicated pregnancy and birth (those in the upper fifth) had similar levels of risk of most complications to those seen in an unselected obstetric population [12, 20-22], and might benefit from different pathways of care compared to all obese women. In this selected group of women with obesity, the observed prevalence of GDM (16\%) was similar to the incidence described in the HAPO study (unselected population) when using the IADPSG criteria (16\%) [12] and the prevalence of preeclampsia (3\%) was similar to that previously reported in lean women (3\%) and lower than previously observed in women with obesity (9\%) [21]. Finally, the prevalence of small for gestational age infants $(10 \%)$ was similar to an unselected population (9-12\%) [20].

Since obese women are currently defined as high risk, a higher AUROC for uncomplicated pregnancy than that achieved by the full model $(0.72,0.68-0.76)$ in this group might be desirable. This also applies to the prediction tool based on clinical factors (AUROC of 0.68 , $0.65-0.71)$. We therefore recommend that the effectiveness of any alternative pathway of care based on these prediction tools should be fully evaluated prior to implementation in clinical practice. Inclusion of other relevant variables, for example, previous obstetric history, weight change from pre-pregnancy to early second trimester or additional biomarkers, may further improve the accuracy of these models. Further studies should also focus on identifying possible causal mechanisms (i.e. biomarkers of good glycaemic control and lower insulin resistance) contributing to uncomplicated pregnancy and birth, which could be amenable to targeted interventions.

Our results have immediate relevance to the choice of place of birth. According to NICE, all women with a
BMI $\geq 35 \mathrm{~kg} / \mathrm{m}^{2}$ should receive recommendation to deliver in an obstetric unit [23]. However, some high risk women still choose to receive care in low risk settings, and therefore clearer direction is needed for shared decision-making. In the Birthplace study, 35\% of 'higher risk' women who planned a home birth were obese $\left(\mathrm{BMI} \geq 30 \mathrm{~kg} / \mathrm{m}^{2}\right)$ [24]. In our study, the rate of spontaneous vaginal delivery in obese women in labour with a BMI $\geq 35 \mathrm{~kg} / \mathrm{m}^{2}$ was $62 \%(57-67 \%)$, similar to that in women with a BMI of $30-35 \mathrm{~kg} / \mathrm{m}^{2}(66 \%, 61-71 \%)$. Importantly, women identified as most likely to have an uncomplicated pregnancy and birth (upper fifth) by our prediction model had a higher rate of spontaneous vaginal delivery of $92 \%$ (87-97\%) following onset of labour. This figure was markedly similar $(90 \%, 86-94 \%)$ in the simpler model including clinical factors alone. In common with our findings, the Birthplace study also identified a subgroup of lower risk women with a BMI $\geq$ $35 \mathrm{~kg} / \mathrm{m}^{2}$ in which the risk for obstetric interventions or maternal adverse outcomes was lower than in nulliparous women of normal BMI (21\% vs. 53\%) [25]. Together, these observations suggest that the current NICE guideline could be modified.

It is generally accepted that obese women should be offered a 75 g OGTT between 24 and 28 weeks' gestation. Lower risk obese women in this study had a GDM prevalence of $16 \%$, similar to the prevalence in an unselected population with the same IADPSG diagnostic criteria [12]. Therefore, it is difficult to suggest a different screening approach for GDM that would be widely accepted. However, the women least likely to have an uncomplicated pregnancy and birth had a GDM prevalence of $43 \%$. These women are likely to benefit from early management or increased surveillance (i.e. prophylactic interventions or early pregnancy OGTT) [26]. Similarly, higher preeclampsia rates were observed for women least likely to have an uncomplicated pregnancy and birth; therefore, these women may benefit from additional blood pressure measurements.

A key strength of this study lies in the prospective collection of detailed clinical, anthropometric and biomarker data related to obesity with contemporaneous data monitoring across all sites. Another strength lies in the assessment of uncomplicated pregnancy and birth, and in the principle of a prediction tool applicable to all pregnancies in obese women rather than one for each outcome (i.e. GDM, preeclampsia, fetal growth restriction). This novel concept simplifies stratification in obese women who are at increased risk of multiple adverse outcomes. A limitation was the number of women without biomarker data $(286 / 1409,27 \%)$. To explore potential selection bias, we assessed the effect size of each clinical factor in the whole study population and in the restricted study cohort (with available biomarkers); 
similar effect sizes were observed in both samples. A 'healthy cohort' effect cannot be excluded; obese women who took part in UPBEAT may have been healthier than the general obese pregnant population, and our prediction model performance would not be generalisable. However, we consider this unlikely as UPBEAT participants had maternal demographic characteristics usually associated with the poorest pregnancy outcomes, being on average 10 months older and with a BMI $0.7 \mathrm{~kg} / \mathrm{m}^{2}$ higher compared to women who declined participation [8]. Other limitations include the low prevalence of some outcomes, such as stillbirth or neonatal death, leading to reduced statistical power. We also acknowledge that external independent validation of our prediction tool is necessary.

\section{Conclusion}

Approximately one-third of the obese women studied had an uncomplicated pregnancy and birth. We have shown that risk stratification could be achieved by a combination of clinical factors and biomarkers. Stratification of risk for an uncomplicated pregnancy is an innovative approach to the management of obese pregnant women, with the potential to improve clinical management and the choices for women as well as to ensure efficient resource allocation. Further studies are needed to explore alternative pathways of care for the sub-groups of obese women who differ according to risk profile for uncomplicated pregnancy and birth.

\section{Additional files}

Additional file 1: Analytical methodologies for the biomarkers measured. (DOCX $13 \mathrm{~kb}$ )

Additional file 2: Sociodemographic characteristics of study population and UPBEAT participants excluded from this analysis. (DOCX $13 \mathrm{~kb}$ )

Additional file 3: Distribution of antenatal and labour complications. (DOCX $38 \mathrm{~kb}$ )

Additional file 4: Factors assessed in relation to uncomplicated pregnancy and birth. (DOCX $15 \mathrm{~kb}$ )

Additional file 5: Pregnancy outcomes according to predicted chance in fifths of uncomplicated pregnancy and birth using the model with clinical factors and HbA1c. (DOCX $15 \mathrm{~kb}$ )

Additional file 6: Sensitivity analysis to assess consistency of association of clinical factors in a restricted sample (women with biomarker data). (DOCX $12 \mathrm{~kb})$

\section{Abbreviations}

AUROC: area under the receiver operating characteristic; BMI: body mass index; GDM: gestational diabetes mellitus; gGT: gamma-glutamyl transferase; HbA1c: haemoglobin A1c; IADPSG: International Association of Diabetes and Pregnancy Study Groups; OGTT: oral glucose tolerance test; SBP: systolic blood pressure; UPBEAT: UK Pregnancies Better Eating and Activity Trial

\section{Acknowledgements}

We thank all staff in the UPBEAT consortium (full list of personnel below) and the participants in the trial for their patience, time, interest and goodwill.
UPBEAT consortium personnel

King's College London/Guy's and St Thomas' NHS Foundation Trust: Lucilla Poston, lead author for consortium (lucilla.poston@kcl.ac.uk), Andrew Shennan, Annette Briley, Claire Singh, Paul Seed, Jane Sandall, Thomas Sanders, Nashita Patel, Angela Flynn, Shirlene Badger, Suzanne Barr, Bridget Holmes, Louise Goff, Clare Hunt, Judy Filmer, Jeni Fetherstone, Laura Scholtz, Hayley Tarft, Anna Lucas, Tsigerada Tekletdadik, Deborah Ricketts, Carolyn Gill, Alex Seroge Ignatian, Catherine Boylen, Funso Adegoke, Elodie Lawley, James Butler, Rahat Maitland, Matias Vieira, Dharmintra Pasupathy. King's College Hospital: Eugene Oteng-Ntim, Nina Khazaezadeh, Jill Demilew, Sile O'Connor, Yvonne Evans, Susan O'Donnell, Ari de la Llera, Georgina Gutzwiller, Linda Hagg.

Newcastle University/Newcastle NHS Foundation Trust: Stephen Robson, Ruth Bell, Louise Hayes, Tarja Kinnunen, Catherine McParlin, Nicola Miller, Alison Kimber, Jill Riches, Carly Allen, Claire Boag, Fiona Campbell, Andrea Fenn, Sarah Ritson, Alison Rennie, Robin Durkin, Gayle Gills, Roger Carr. Glasgow University and Greater Clyde Health Board: Scott Nelson, Naveed Sattar, Therese McSorley, Hilary Alba, Kirsteen Paterson, Janet Johnston, Suzanne Clements, Maxine Fernon, Savannah Bett, Laura Rooney, Sinead Miller, Paul Welsh, Lynn Cherry.

Central Manchester Hospitals Foundation Trust: Melissa Whitworth, Natalie Patterson, Sarah Lee, Rachel Grimshaw, Christine Hughes, Jay Brown. City Hospital Sunderland: Kim Hinshaw, Gillian Campbell, Joanne Knight. Bradford Royal Infirmary: Diane Farrar, Vicky Jones, Gillian Butterfield, Jennifer Syson, Jennifer Eadle, Dawn Wood, Merane Todd.

St George's NHS Trust, London: Asma Khalil, Deborah Brown, Paola Fernandez, Emma Cousins, Melody Smith.

University College London: Jane Wardle, Helen Croker, Laura Broomfield (Weight Concern - Registered Charity No. 1059686৫).

University of Southampton: Keith Godfrey, Sian Robinson, Sarah Canadine, Lynne Greenwood.

Trial Steering Committee: Catherine Nelson-Piercy, Stephanie Amiel, Gail Goldberg, Daghni Rajasingham, Penny Jackson, Sara Kenyon, Patrick Catalano.

\section{Funding}

This study was supported by National Institute for Health Research (NIHR; RP-PG-0407-10452; https://www.nihr.ac.uk), Chief Scientist Office Scottish Government Health Directorates (Edinburgh; CZB/4/680; http:// www.cso.scot.nhs.uk), Guys and St Thomas' Charity (1060508; https:// www.gsttcharity.org.uk), Tommy's Charity (SCO39280; https:// www.tommys.org), Medical Research Council UK (MR/L002477/1; https:// www.mrc.ac.uk), European Research Council under the European Union's Seventh Framework Programme (FP7/2007-2013)/ERC grant agreement 669545 (https://ec.europa.eu/research/fp7/index_en.cfm) and the US National Institute of Health (R01 DK10324; https://www.nih.gov). It was also supported by the NIHR Biomedical Research Centre at Guy's and St Thomas' NHS Foundation Trust and Kings College London, and NIHR Biomedical Research Centre at the University Hospitals Bristol NHS Foundation Trust and the University of Bristol. MCV receives support from Coordenadoria de Aperfeiçoamento de Pessoal de Ensino Superior (CAPES; BEX 9571/13-2; http://www.capes.gov.br). SLW receives support from Diabetes UK (14/0004849; https://www.diabetes.org.uk). DAL works in a Unit that receives support from the UK Medical Research Council (MC_UU_1201/5) and she, LP and JS are NIHR Senior Investigators (NF-SI-016610196, NF-SI-0512-10104, and NF-SI-0514-10145, respectively). PTS, ALB and JS are supported by the NIHR Collaboration for Leadership in Applied Health Research and Care South London at King's College Hospital NHS Foundation Trust. DP has received support from NIHR and Tommy's Charity. The views expressed are those of the authors and not necessarily those of the NHS, the $\mathrm{NIHR}$ or the Department of Health. The funders and the sponsor played no role in the design and conduct of the study, collection, management, analysis and interpretation of the data, preparation, review or approval of the manuscript, or decision to submit the manuscript for publication.

\section{Availability of data and materials}

The datasets generated and/or analysed during the current study are not publicly available due to consent obtained from participants, which did not include permission to publicly share their data. The data that support the findings of this study are available on reasonable request from the senior author (DP), subject to approval of the UPBEAT Scientific Committee. 


\section{Authors' contributions}

All listed authors meet the requirements for authorship. PTS, ALB, JS, NS, SMN and LP contributed to the original UPBEAT trial design. MCV, SLW, NP, PTS, ALB, NS, SMN, DAL, LP and DP designed this component of the UBEAT study. MCV, SLW, NP, PTS and DP performed and interpreted the statistical analysis. ALB, JS, PW, NS, SMN, DAL and LP reviewed the data. MCV, LP and DP wrote the manuscript. All authors had full access to the data, critically reviewed the manuscript, approved the final version, and agreed to be accountable for all aspects of the work.

\section{Authors' information}

Not applicable.

\section{Ethics approval and consent to participate}

Ethical approval was obtained from the Brighton Research Ethics Committee and all women provided informed written informed consent prior to entering the study (UK integrated research application system, reference 09/H0802/5).

\section{Consent for publication}

Not applicable.

\section{Competing interests}

All authors have completed the ICMJE uniform disclosure form at http:/ uww.icmje.org/downloads/coi_disclosure.pdf and declare: for the submitted work, MCV had financial support from Coordenadoria de Aperfeiçoamento de Pessoal de Ensino Superior (CAPES); SLW had financial support from Diabetes UK; NP, PTS, ALB, LP and DP had financial support from Tommy's Charity; PTS, ALB, JS, NS, SMN, DAL, LP and DP had financial support from Medical Research Council (MRC) and National Institute of Health Research (NHHR); no financial relationships with any organisations that might have an interest in the submitted work in the previous 5 years; and no other relationships or activities that could appear to have influenced the submitted work.

\section{Publisher's Note}

Springer Nature remains neutral with regard to jurisdictional claims in published maps and institutional affiliations.

\section{Author details}

'Department of Women and Children's Health, School of Life Course Sciences, Faculty of Life Sciences and Medicine, King's College London, London SE1 7EH, UK. ${ }^{2}$ Núcleo de Formação Específica em Ginecologia e Obstetrícia, Escola de Medicina, Pontifícia Universidade Católica do Rio Grande do Sul, Porto Alegre 90610-000, Brazil. ${ }^{3} \mathrm{NIHR}$ Biomedical Research Centre at Guy's and St Thomas' NHS Foundation Trust and King's College London, London SE1 7EH, UK. ${ }^{4}$ Institute of Cardiovascular and Medical Sciences, University of Glasgow, Glasgow G12 8TA, UK. ${ }^{5}$ School of Medicine, University of Glasgow, Glasgow G4 OSF, UK. ${ }^{6}$ MRC Integrative Epidemiology Unit and School of Social and Community Medicine, University of Bristol, Bristol BS8 2BN, UK. ${ }^{7} \mathrm{NIHR}$ Biomedical Research Centre at University Hospitals Bristol NHS Foundation Trust and University of Bristol, Bristol BS8 2BN, UK. ${ }^{8}$ Department of Women and Children's Health, Women's Health Academic Centre KHP, St. Thomas' Hospital, Westminster Bridge Road, 10th Floor North Wing, London SE1 7EH, UK.

Received: 24 May 2017 Accepted: 11 October 2017 Published online: 03 November 2017

\section{References}

1. Poston L, Caleyachetty R, Cnattingius S, Corvalan C, Uauy R, Herring S, et al. Preconceptional and maternal obesity: epidemiology and health consequences. Lancet Diabetes Endocrinol. 2016;4(12):1025-36.

2. Devlieger R, Benhalima K, Damm P, Van Assche A, Mathieu C, Mahmood T, et al. Maternal obesity in Europe: where do we stand and how to move forward?: A scientific paper commissioned by the European Board and College of Obstetrics and Gynaecology (EBCOG). Eur J Obstet Gynecol Reprod Biol. 2016;201:203-8.

3. Morgan KL, Rahman MA, Macey S, Atkinson MD, Hill RA, Khanom A, et al. Obesity in pregnancy: a retrospective prevalence-based study on health service utilisation and costs on the NHS. BMJ Open. 2014;4(2):e003983.

4. NHS England. Five Year Forward View. 2016. https://www.england.nhs.uk/ wp-content/uploads/2014/10/5yfv-web.pdf, Accessed on 24 Nov 2016.
5. National Maternity Review. Better Births - Improving Outcomes of Maternity Services in England. 2016. https://www.england.nhs.uk/wp-content/uploads/ 2016/02/national-maternity-review-report.pdf. Accessed on 24 Nov 2016.

6. Chappell LC, Seed PT, Myers J, Taylor RS, Kenny LC, Dekker GA, et al. Exploration and confirmation of factors associated with uncomplicated pregnancy in nulliparous women: prospective cohort study. BMJ. 2013;347:f6398.

7. Poston L, Briley AL, Barr S, Bell R, Croker H, Coxon K, et al. Developing a complex intervention for diet and activity behaviour change in obese pregnant women (the UPBEAT trial); assessment of behavioural change and process evaluation in a pilot randomised controlled trial. BMC Pregnancy Childbirth. 2013;13:148.

8. Poston L, Bell R, Croker H, Flynn AC, Godfrey KM, Goff L, et al. Effect of a behavioural intervention in obese pregnant women (the UPBEAT study): a multicentre, randomised controlled trial. Lancet Diabetes Endocrinol. 2015; 3(10):767-77.

9. Moons KG, Royston P, Vergouwe $Y$, Grobbee DE, Altman DG. Prognosis and prognostic research: what, why, and how? BMJ. 2009;338:b375.

10. Cole TJ, Freeman JV, Preece MA. British 1990 growth reference centiles for weight, height, body mass index and head circumference fitted by maximum penalized likelihood. Stat Med. 1998;17(4):407-29.

11. Report of the National High Blood Pressure Education Program Working Group on High Blood Pressure in Pregnancy. Am J Obstet Gynecol. 2000;183(1):S1-S22.

12. IADPSGC. International association of diabetes and pregnancy study groups recommendations on the diagnosis and classification of hyperglycemia in pregnancy. Diabetes Care. 2010;33(3):676-82.

13. Harrison GGBE, Carter JEL, Johnston FE, Lohman TG, Pollock ML, Roche AF, Wilmore J. Skinfold thicknesses and measurement technique. In: Lohman TGRA, Martorell R, editors. Anthropometric Standardization Manual. Champaign, IL: Human Kinetics Books; 1991. p. 55-70.

14. Grobman WA, Stamilio DM. Methods of clinical prediction. Am J Obstet Gynecol. 2006;194(3):888-94.

15. Ivanescu AE, Li P, George B, Brown AW, Keith SW, Raju D, et al. The importance of prediction model validation and assessment in obesity and nutrition research. Int J Obes (Lond). 2016;40(6):887-94.

16. Collins GS, Reitsma JB, Altman DG, Moons KG. Transparent reporting of a multivariable prediction model for individual prognosis or diagnosis (TRIPOD): the TRIPOD statement. BMJ. 2015;350:g7594.

17. World Health Organization. WHO Recommendations on Antenatal Care for a Positive Pregnancy Experience. Geneva: WHO; 2016.

18. National Institute for Health and Clinical Excellence. Antenatal Care for Uncomplicated Pregnancies. NICE guideline (CG62). 2008. https://www.nice. org.uk/guidance/cg62. Accessed on 20 Mar 2017.

19. ACOG. Practice Bulletin No 156: Obesity in Pregnancy. Obstet Gynecol. 2015;126(6):e112-26.

20. Gardosi J, Francis A. Adverse pregnancy outcome and association with small for gestational age birthweight by customized and population-based percentiles. Am J Obstet Gynecol. 2009;201(1):28. e1-8.

21. Vieira MC, Poston L, Fyfe E, Gillett A, Kenny LC, Roberts $C T$, et al. Clinical and biochemical factors associated with preeclampsia in women with obesity. Obesity (Silver Spring). 2017;25(2):460-7.

22. Royal College of Obstetricians and Gynaecologists. Patterns of Maternity Care in English NHS Trusts 2013/2014. 2016. https://www.rcog.org.uk/ globalassets/documents/guidelines/research\%2D-audit/maternity-indicators2013-14_report2.pdf. Accessed on 30 June 2016.

23. National Institute for Health and Clinical Excellence. Intrapartum Care for Healthy Women and Babies. NICE guideline (CG190). 2014. https://www. nice.org.uk/guidance/cg190. Accessed on 30 June 2016.

24. Li Y, Townend J, Rowe R, Brocklehurst $P$, Knight $M$, Linsell $L$, et al. Perinatal and maternal outcomes in planned home and obstetric unit births in women at 'higher risk' of complications: secondary analysis of the Birthplace national prospective cohort study. BJOG. 2015;122(5):741-53.

25. Hollowell J, Pillas D, Rowe R, Linsell L, Knight M, Brocklehurst P. The impact of maternal obesity on intrapartum outcomes in otherwise low risk women: secondary analysis of the Birthplace national prospective cohort study. BJOG. 2014;121(3):343-55.

26. White SL, Lawlor DA, Briley AL, Godfrey KM, Nelson SM, Oteng-Ntim E, et al. Early antenatal prediction of gestational diabetes in obese women: development of prediction tools for targeted intervention. PLoS One. 2016;11(12):e0167846. 